\title{
Optimização da perviabilidade do enxerto venoso na revascularização miocárdica: compreensão da fisiopatologia, novas drogas e avanços técnicos
}

\author{
Danton R. Rocha LOURES*, Edison José RIBEIRO**, Leonardo Andrade MULINARI***, \\ Roberto Gomes de CARVALHO ${ }^{* * *}$, Rui Sequeira de ALMEIDA**** ${ }^{* *}$ Marcelo Laneza FELÍCIO***
}

RBCCV 44205-525

Loures D R R, Ribeiro E J, Mulinari L A, Carvalho R G, Almeida R S, Felício M L - Optimização da perviabilidade do enxerto venoso na revascularização miocárdica: compreensão da fisiopatologia, novas drogas e avanços técnicos. Rev Bras Cir Cardiovasc 2001; 16(1): 14-9.

RESUMO: Objetivo: Baseado nos modernos conceitos do comportamento endotelial do enxerto venoso, o objetivo deste trabalho foi identificar quais os principais fatores de obstrução no período imediato da cirurgia, quais e quando ocorrem as modificações morfológicas tardias e, finalmente, apontar os recursos aplicáveis para optimizar a perviabilidade imediata e tardia dos enxertos venosos.

Casuística e Métodos: No período de 1971 a 1998 foram operados 3116 pacientes de revascularização miocárdica. Estes procedimentos foram analisados em três grupos- grupo I onde somente se empregou veia safena; grupo II onde somente se empregou artéria torácica interna e grupo III onde se empregou a associação de veia safena e artéria torácica interna. A incidência de reoperações foi analisada procurando identificar a perviabilidade dos diferentes enxertos e seus resultados. Foi realizada uma análise estatística empregando-se o teste de Qui-quadrado para averiguar a existência de diferenças de reoperados e mortalidade entre os três grupos.

Resultados: $O$ índice de mortalidade imediata variou de 1 a $7 \%$ no grupo I para 3,8 \% no grupo II demonstrando diferença estatisticamente significativa $(p=0,0401)$. Foram reoperados 255 pacientes, sendo $3,8 \%$ no grupo II e $8,1 \%$ no grupo I demonstrando diferença estatisticamente significativa $(p=0,0094)$.

Conclusões: Os resultados imediatos com os enxertos venosos dependem do manuseio cirúrgico, retirada. e preparo da veia, confecção das anastomoses e qualidade das artérias coronárias. Os resultados tardios entre o quinto e décimo anos sofrem a influência do espectro da aterosclerose. Os enxertos venosos representam maior incidência de reoperações quando comparados com os enxertos arteriais. Os enxertos venosos podem aumentar o grau de perviabilidade através da limitação do manuseio da veia safena, emprego de drogas como verapamil, triglicerina, papaverina, aprotinina, da profilaxia da aterosclerose e da uniformização da luz das veias irregularmente dilatadas através de enxertos tubulares híbridos.

DESCRITORES: Revascularização miocárdica, métodos. Artéria mamária, transplante. Veia safena, transplante. Artérias mamárias fisiopatologia. Veia safena, fisiopatologia.

Trabalho multicêntrico realizado no Serviço de Cirurgia Torácica e Cardiovascular da Universidade Federal do Paraná, no Serviço de Cirurgia Cardiovascular do Hospital Universitário Evangélico de Curitiba e no Serviço de Cirurgia Cardíaca do Hospital Policlínica de Cascavel do Paraná, PR, Brasil. Recebido para publicação em novembro de 2000.

*Da Universidade do Paraná, do Hospital Universitário Evangélico e do Hospital Policlínica de Cascavél

**Do Hospital Universitario Evangélico.

${ }^{* * *}$ Da Universidade do Paraná.

****Do Hospital Policlínica de Cascavel.

Endereço para correspondência Danton R. Rocha Loures. Rua Augusto Stellfeld, 2088, Curitiba, PR, Brasil, CEP 80730-150. Tel. (41) 222-7714. Fax. (41) 264.5872 e 360.1800 (Hospital de Clínicas). Celular: 99727413. 
Loures D R R, Ribeiro E J, Mulinari L A, Carvalho R G, Almeida R S, Felício M L - Optimização da perviabilidade do enxerto venoso na revascularização miocárdica: compreensão da fisiopatologia, novas drogas e avanços técnicos. Rev Bras Cir Cardiovasc 2001; 16(1): 14-9.

\section{INTRODUÇÃO}

Desde 1977, quando FAVALORO et al. (1) empregaram, pela primeira vez, a veia safena autológa como um conduto arterial na revascularizado coronária, este enxerto venoso tem se consagrado como o mais largamento empregado não obstante a sua perviabilidade a longo prazo tenha sido apontada como um fator critico, principalmente quando se compara com a longevidade dos enxertos arteriais (2).

O comportamento morfológico dos enxertos venosos podem sofrer influência de falhas no manuseio cirúrgico, erros técnicos na confecção das pontes, hiperplasia miointimal, fibrose, trombose e progressiva aterosclerose ${ }^{(3,4)}$.

O grau destas alterações morfológicas e a significância funcional têm sido atribuídos a muitos fatores, entre os quais se destacam a pressão intraluminal, isquemia na parede do enxerto, depósito de fibrina e trombose, e a resposta endotelial quando agredida pela isquemia, trauma ou ambos (5).

\section{OBJETIVO}

O objetivo deste trabalho foi de analisar a experiência de nosso grupo de 3116 casos de revascularização miocárdica e sob a luz dos modernos conceitos do comportamento endotelial do enxerto venoso identificar quais os principais fatores de obstrução no período imediato da cirurgia, quais e quando ocorrem, as modificaq6es morfológicas tardias e, finalmente, apontar os recursos aplicáveis para otimizar a perviabilidade imediata e tardia dos enxertos venosos.

\section{Fisiopatologia}

CARREL \& GUTHRIE (6) descreveram, em 1906, o espessamento intimal das veias implantadas em cães, e concluíram que as veias colocadas na circulação arterial tinham uma forte tendência para assumir o cateter de uma artéria. Dois anos mais tarde, o mesmo CARREL (7) descreveu quatro alterações características nas veias usadas como artérias nas veias periféricas, tais como, (a) espessamento intimal, (b) espessamento da adventícia, (c) perda do terço interno da camada média e (d) perda da elasticidade da veia transformandoa num tubo fibroso.

UNNI et al. (8) estudaram 62 enxertos de 40 pacientes em períodos variáveis de um até 28 meses após a cirurgia. Os achados mais precoces identificados foram a migração de elementos do sangue para dentro da íntima e migração de leucócitos dentro da média e intima, presumivelmente em resposta ao dano endotelial.

ANGELINI et al. (9), demonstraram, experimentalmente em porcos, que as veias safenas submetidas à distensão de suas paredes com pressões de até $600 \mathrm{~mm} \mathrm{Hg}$ e posteriormente implantadas na circulação arterial comprovam um dano da média e endotelial com associação de uma aumentada adesão de plaquetas e leucócitos com redução precoce de sua perviabilidade.

FAULKNER et al. (10), estudaram o efeito do índice de fluxo sangüíneo através dos enxertos venosos e interpretaram que a proliferação intimal subendotelial ocorrida nas porções distal e proximal poderiam indicar a turbulência de fluxo causada pelas anastomoses como causa de problemas técnicos das anastomoses cirúrgicas.

SPRAY \& ROBERTS (11) mencionam, além dos problemas técnicos de cirurgia, outras causas de obstrução imediata dos enxertos, tais como, o estreitamento distal da artéria coronária, anastomose em cima de placa ateromatosa, dissecção distal da artéria coronária e, finalmente, desproporção veia safena/artéria coronária.

BRODY et al. (12), realizaram estudos experimentais em cães e observaram alterações tardias nos enxertos venosos tais como hipertrofia e morte do músculo liso da camada media, transformação fibroblástica resultando numa fibrose medial e intimal. Sugerem ainda que estas alterações são produzidas pela transformação dos miócitos da medial em resposta à injúria ou ainda pela mudança de seu estado nutricial. A resposta do miócito pode ser mediada pela elevada tensão do oxigênio na parede com conseqüentes efeitos no fosfolípides ou pela presença de fatores plasmáticos ou produtos sangüíneos como um resultado do recurrente dano endotelial.

\section{CASUÍSTICA E MÉTODOS}

No período de setembro, de 1971 a agosto de 1998 foram operados 3116 casos de revascularização miocárdica com circulação extracorpórea (CEC).

Os procedimentos cirúrgicos foram analisados nos grupos dos enxertos venosos isolados, enxertos arteriais isolados e enxertos venosos e arteriais associados, respectivamente nos diferentes períodos de 1971-80, 1981-90 e 1990-98.

A incidência de reoperações foi analisada procurando identificar a perviabilidade dos diferentes enxertos e seus resultados.

Foi analisada, neste material, uma inovação no tratamento de veias ectásicas e varicosas em- 
Loures D R R, Ribeiro E J, Mulinari L A, Carvalho R G, Almeida R S, Felício M L - Optimização da perviabilidade do enxerto venoso na revascularização miocárdica: compreensão da fisiopatologia, novas drogas e avanços técnicos. Rev Bras Cir Cardiovasc 2001; 16(1): 14-9.

TABELA 1

IDADE E SEXO

\begin{tabular}{|c|c|c|c|}
\hline Idade & 22 - 85 anos & Média 52,4 anos & \\
\hline Sexo & $\begin{array}{l}\text { Masculino } \\
\text { Feminino }\end{array}$ & $\begin{array}{l}2131 \text { casos } \\
9885 \text { casos }\end{array}$ & $\begin{array}{l}68,54 \% \\
3,1 \%\end{array}$ \\
\hline
\end{tabular}

TABELA 2

ANÁLISE DE MORTALIDADE

\begin{tabular}{ll}
\hline Revascularização isolada de artéria mamária & $4 / 131=3,0 \%$ \\
Revascularização isolada de ponte de safena & $32 / 1838=1,7 \%\left({ }^{*}\right)$ \\
Revascularização associada VS e AMI & $35 / 1147=3,1 \%$ \\
${ }^{*}$ RM + ventriculectomia & $12 / 132=9,1 \%$ \\
${ }^{*}$ RM + valva & $15 / 112=13,4$ \\
${ }^{*}$ RM + outros procedimentos & $5 / 38=15,8 \%$ \\
\hline
\end{tabular}

(*) Em 6 casos foram empregados o biocompound graft.

TABELA 3

\begin{tabular}{|c|c|c|c|}
\hline \multicolumn{4}{|c|}{ INCIDÊNCIA DE REOPERAÇÕES NOS TRÊS GRUPOS } \\
\hline & $\begin{array}{c}\text { GRUPO I } \\
\text { VS ISOLADA }\end{array}$ & $\begin{array}{c}\text { GRUPO II } \\
\text { AMI ISOLADA }\end{array}$ & $\begin{array}{c}\text { GRUPO III } \\
\text { VS AMI ASSOCIADA }\end{array}$ \\
\hline $1^{\mathrm{a}}$ reoperação & 132 & 5 & 96 \\
\hline $2^{\mathrm{a}}$ reoperação & 14 & - & 6 \\
\hline $3^{\mathrm{a}}$ reoperação & 2 & - & - \\
\hline TOTAL & $\begin{array}{l}148 \\
8,1 \%\end{array}$ & $\begin{array}{l}5 \\
3,8 \%\end{array}$ & $\begin{array}{l}102 \\
7,0 \%\end{array}$ \\
\hline
\end{tabular}

pregando-se um composto biológico e um tubo microfilamentar metálico (biocompound graft) idealizado por ZURBRUGG \& HETZER (12) da Universidade de Berlin (Figura 1).

\section{Análise Estatística}

Testou-se a hipótese nula de independência entre a necessidade de reoperação e o tipo de procedimento prévio entre os três grupos, versus a hipótese alternativa de dependência entre estas variáveis.

O teste estatístico adotado foi o de Qui-quadrado, para a análise de mortalidade não foi possível a realização do teste de independência. Desta forma, testou-se a hipótese nula de proporções iguais para. os dois tipos de revascularização sob comparação, versus a hipótese alternativa. de proporções diferentes.

\section{RESULTADOS}

A idade dos pacientes variou de 22 a 85 anos com idade media de 52,4 anos e o sexo masculino foi predominante em $68,4 \%$ (Tabela 1 ).

O índice de mortalidade imediata variou de 1,7\% na revascularização com ponte de veia safena, até $15,8 \%$ nos procedimentos associados e complexos (Tabela 2).

Foram reoperados 255 casos de pacientes, sendo $3,8 \%$ com artéria torácica interna isolada (grupo II) e $8,1 \%$ com pontes de veia safena isolada (Tabela 3 ).

O estudo estatístico na comparação dos 3 grupos onde se testou a hipótese nula de proporções iguais de necessidade de reoperação, versus a hipótese alternativa de proporção menor de reoperação para o procedimento de artéria torácica interna indicou a rejeição da hipótese de proporções iguais ao nível de significância de 5\% 
Loures D R R, Ribeiro E J, Mulinari L A, Carvalho R G, Almeida R S, Felício M L - Optimização da perviabilidade do enxerto venoso na revascularização miocárdica: compreensão da fisiopatologia, novas drogas e avanços técnicos. Rev Bras Cir Cardiovasc 2001; 16(1): 14-9.
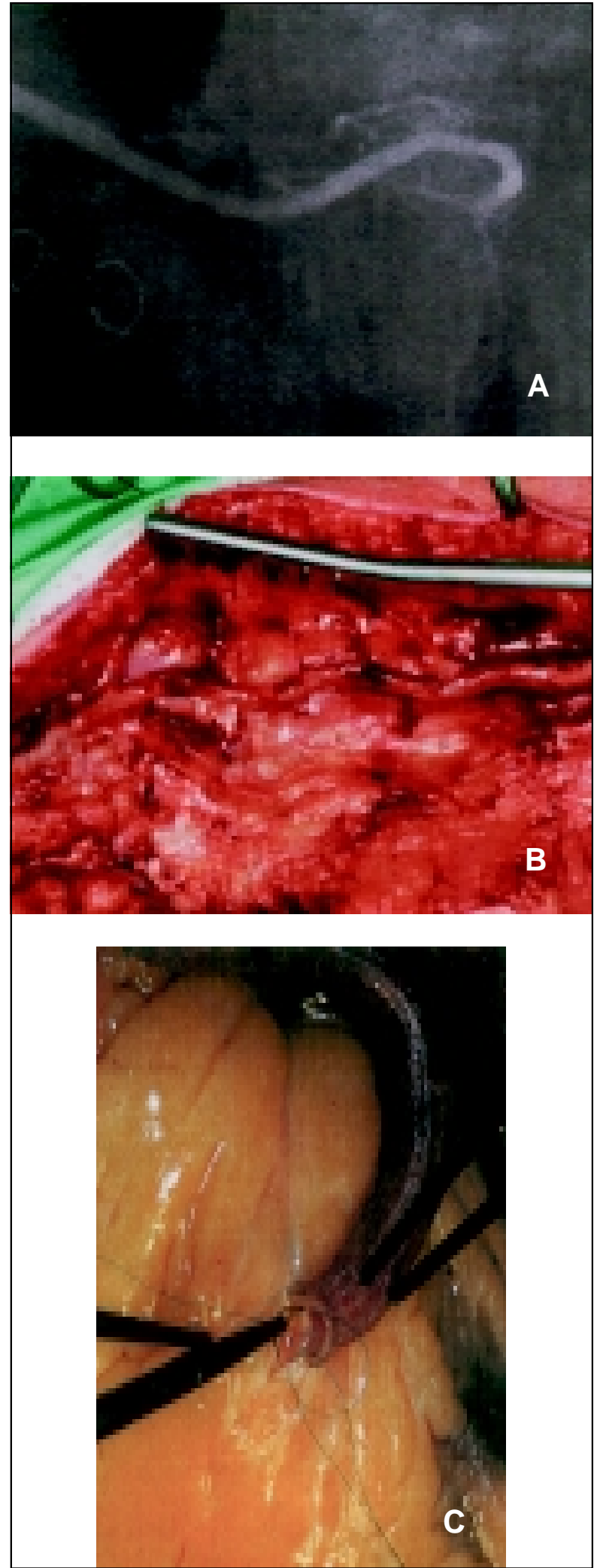

Fig. 1 - Biocompound graft

a) vista intraoperatória de veia varicosa dissecada;

b) manuseio cirúrgico facilitado;

c) controle angiográfico com 12 meses de pós-operatório $(p=0,0401)$ quando comparada com o grupo de veia safena isolada; o mesmo ocorrendo, quando comparada com o grupo de veia safena e artéria torácica interna associada ( 0 0,0235). Quando se comparou estes dois últimos grupos entre si (grupo I e III) não houve diferença estatisticamente significativa $(p=0,4200)$.

O estudo estatístico na comparação dos três grupos onde se testou. a hipótese nula de proporções iguais de óbito, versus a hipótese alternativa de proporção menor de óbito, o resultado revelou que o grupo I (revascularização com ponte de safena isolada) apresentou menor mortalidade quando, comparada com o grupo III (veia safena e, artéria torácica interna associadas) onde $p=0,0094$. E não houve diferença entre os demais grupos comparados entre si.

\section{COMENTÁRIOS}

As modificações morfológicas e obstruções são comuns após a cirurgia de revascularização miocárdica com enxerto venoso e aumentam com o tempo.

FITZGIBBON et al. (14), acompanharam angiograficamente 5065 enxertos venosos e arteriais por um período de 25 anos e observaram que ambos enxertos apresentaram uma correlação estreita e direta entre tempo de evolução e índice de obstrução, sendo mais acentuada nos enxertos venosos.

Observando nossos resultados em 27 anos de experiência encontramos os mesmos achados, pois as reoperações no grupo I (veia safena isolada) foi 2,1 vezes maior que no grupo II (artéria mamaria isolada sendo estatisticamente significativa $(p=$ 010401). CAMPEAU et al. $(15,16)$ interpretaram que as modificações morfológicas dos enxertos de veia safena são aparentemente decorrentes da aterosclerose e parece ser infreqüente nos primeiros 7 anos. O acompanhamento clínico dos três grupos de nosso estudo revelou serem semelhantes nos primeiros 5 anos.

SOYOMBO et al. (17), estudando a elevação do monofosfato de adenosina ou guanosina cíclico durante o manuseio cirúrgico, procuraram correlacionar estes achados com o espessamento intimal da parede dos enxertos venosos. Demonstraram uma diminuição de $40-80 \%$ do espessamento intimal quando os níveis destes monofosfatos endógenos estavam numa. concentração inferior a $0,1 \mathrm{mmol} / \mathrm{L}$.

Na nossa experiência há uma correlação imediata entre os novos médicos residentes e uma elevação na incidência de obstrução imediata dos enxertos 
Loures D R R, Ribeiro E J, Mulinari L A, Carvalho R G, Almeida R S, Felício M L - Optimização da perviabilidade do enxerto venoso na revascularização miocárdica: compreensão da fisiopatologia, novas drogas e avanços técnicos. Rev Bras Cir Cardiovasc $2001 ; 16(1): 14-9$.

venosos. A interpretação deste fato tem sido atribuída à menor experiência destes jovens cirurgiões no manuseio durante a retirada da veia safena.

Para amenizar o índice de obstrução imediata dos enxertos venosos, WENDEL et al. (18) recomendam o emprego de altas. doses de aprotinina quando os índices de troponina T estiverem elevados no pré-operatório ou em casos complexos onde se prevê um manuseio cirúrgico mais laborioso.

BRAUNER et al. (19) recomendam a administração sobre o enxerto venoso de verapamil visando inibir a proliferação neointimal de células musculares lisas e melhorar as anormalidades vasomotoras. Acreditam os autores que possa inibir estes fenômenos entre 28 e $45,0 \%$.

ZURBRUGG \& HETZER (13) recomendam, o emprego de uma prótese vascular híbrida (biocompound graft), para as situações especiais onde os enxertos venosos sejam varicosos. Numa experiência de seu grupo de 200 casos associada a outra multicêntrica internacional com mais de 650 casos, observaram, num acompanhamento tardio de mais de 3 anos, um índice de patenticidade de 92,9\%.

$\mathrm{Na}$ nossa experiência as razões destes resultados favoráveis estão baseados na transformação das veias varicosas em enxertos com diâmetro uniforme, diminuição da tensão na parede, diminuição dos acotovelamentos e facilidade no manuseio cirúrgico.

\section{CONCLUSÕES}

Os resultados imediatos com os enxertos venosos dependem do manuseio cirúrgico, retirada e preparo da veia, confecção das anastomoses e qualidade das artérias coronárias.

- Os resultados tardios entre o quinto e décimo ano sofrem a influência do espectro da aterosclerose.

- Os enxertos venosos representam maior incidência de reoperações quando comparados com os enxertos arteriais.

- Os enxertos venosos podem aumentar o grau de perviabilidade através da limitação do manuseio da veia safena, emprego de drogas como veraparnil, triglicerina, papaverina, aprotinina, da profilaxia da aterosclerose e da. uniformização da luz das veias irregularmente dilatadas através de enxertos tubulares híbridos.

RBCCV 44205-525

Loures D R R, Ribeiro E J, Mulinari L A, Carvalho R G, Almeida R S, Felício M L - The optimization of the venous grafts patency in myocardial revascularization: understanding of the physiopatology, new drugs and technical advances. Rev Bras Cir Cardiovasc 2001; 16(1): 14-9.

RESUMO: Background: This study analyzes the experience of 3116 cases of coronary artery by-pass grafting revascularization of our group and define the percentage of early and late patency of the venous grafts. We also look this issue under the new concepts of endothelial reaction, influence of various drugs and improvement of surgical techniques.

Material and Methods: From September 1971 until August 1998, 3116 cases of myocardial revascularization with cardiopulmonary by-pass were operated in our service. The surgical procedures were analyzed separately regarding the type of grafts used in each case; exclusively venous grafts, exclusively arterial grafts and venous and combined arterial grafts. We also made the analysis in three different periods, 1971-80, 1981-90 and 1991-98. The incidence of reoperation was analyzed trying to identify the patency of the different types of grafts and their results.

Results: The age of the patients varied from 22 to 85, with a mean of 52.4 years, male sex was predominant with $68.4 \%$. The mortality varied from $1.7 \%$ for vein grafts revascularization to $15.8 \%$ when associated complex procedures were done. Of the 3116 patients, 255 were reoperated, being $3.8 \%$ with isolated internal thoracic artery and $8.1 \%$ isolated vein graft.

Conclusions: a) The immediate result of vein grafts patency was dependent upon the surgical handling during vein harvesting and preparations, care during anastomosis and quality of coronary arteries. b) The late results in the $5^{\text {th }}$ and $10^{\text {th }}$ year were influenced by the spectrum of the arteriosclerosis. c) Patients with vein grafts represent the majority of reoperations when compared with those who received arterial grafts. d) There is a possibility of increasing vein grafts patency by a better handling of the graft, use of drugs such as calcium blockers, papaverin, aprotinin, nitrates and the prophylaxis of arteriosclerosis.

DESCRIPTORS: Myocadial revascularization, methods. Mammary arteries, transplantation. Saphenous vein, transplantation. Mammary arteries, physiopathology. Saphenous vein, physiopathology. 
Loures D R R, Ribeiro E J, Mulinari L A, Carvalho R G, Almeida R S, Felício M L - Optimização da perviabilidade do enxerto venoso na revascularização miocárdica: compreensão da fisiopatologia, novas drogas e avanços técnicos. Rev Bras Cir Cardiovasc 2001; 16(1): 14-9.

\section{BIBLIOGRAFIA}

1 Favaloro R G, Effler D B, Groves L K, Sones Jr. FM, Fergusson D J - Myocardial revascularization by internal mammary artery implant procedures: clinical experience. J Thorac Cardiovasc Surg 1967; 54: 359-70.

2 Galbut D L, Traad E A, Dorman M J et al. - Seventeenyear experience with bilateral internal mammary artery grafts. Ann Thorac Surg 1990; 49: 195-201.

3 FitzGibbon G M, Burton J R, Leach A J - Coronary bypass graft fate: angiographic grading of 1400 consecutive grafts early operation and of 1132 after one year. Circulation 1978; 57: 1070-4.

4 Grondin C M - Graft disease in patients with coronary bypass grafting: why does it start? Where do we stop? (Editorial). J Thorac Cardiovasc Surg 1986; 92: 323-9.

5 FitzGibbon G M, Leach A J, Kafka H P, Keon W J Coronary bypass graft fate: long term angiographic study. J Am Coll Cardiol 1991; 17: 1075-80.

6 Carrel A \& Guthrie C C - Results of biterminal transplantation of veins, Am J Med Sci 1906; 132: 415.

7 Carrel A - Results of transplantation of blood vessels, organs, and limbs, JAMA 1908; 51:1662.

8 Unni K K, Kottke B A, Titus J L, Frye RL, Wallace RB, Brown $A L$ - Pahologic changes in aortocoronary saphenous vein grafts. Am J Cardiol 1974; 34: 526-32.

9 Angelini G D, Bryan A J, Williams H M J, Morgan R, Newby A C - Distention promotes platelet and leukocyte adhesion and reduces short-term patency in pig arteriovenous bypass grafts. J Thorac Cardiovasc Surg 1990; 99: 433-9.

10 Faulkner S L, Fisher R D, Conkle D M, Page DL, Bender HW - Effect of blood flow rate on subendothelial proliferation in venous autografts used as arterial substitutes. Circulation 1975; 52 (Suppl 2): 63-72.

11 Spray $T L$ \& Roberts W C - Changes in saphenous veins used as aortocoronary bypass grafts. Am J Heart 1977; 94: 500-16.
12 Brody W F, Angel W W, Kosek J C - Histologic fate of the venous coronary artery bypass in dogs. $A m \mathrm{~J}$ Pathol 1972; 66: 111-30.

13 Zurbrugg H R \& Hetzer R - The issue of cost in innovations in aortocoronary bypass surgery. In: Dohnnann $P$, Henne-Bruns D, Kremer B (eds): Surgical efficiency and economy (SEE), Georg Thierne Verlag, Germany 1997: 258.

14 FitzGibbon G M, Kafka H P, Leach A J, Keon W J, Hooper $G D$, Burton J R - Coronary bypass graft fate and patient outcome: angiographic follow-up of 5,065 grafts related to survival and reoperation in 1,388 patients during 25 years. J Am Coll Cardiol 1996; 28: 616-26.

15 Campeau L, Enjalbert M, Lesperance J, Vaislic C, Grondin C M, Bourassa M G - Atherosclerosis and late closure of aortocoronary saphenous vein grafts: sequential. angiographic studies at 2 weeks, I year, 5 to 7 years, and 10 to 12 years after surgery. Circulation 1983; 68(3 Pt 2): 1-7.

16 Campeau L, Enjalbert M, Lesperance J et al. - The relation of risk factors, to the development of atherosclerosis in the saphenous-vein bypass grafts and the progression of disease in the native circulation: a study 10 years after aortocoronary bypass surgery. N Engl J Med 1984; 311: 1329-32.

17 Soyombo A A, Angelini G D, Newby A C - Neointima formation is promoted by surgical preparation and inhibited by cyclic nucleotides in human saphenous vein organ cultures. J Thorac Cardiovasc Surg 1995; 109: 2-12.

18 Wendel H P, Heller W, Michel J et al. - Lower cardiac troponin $\mathrm{T}$ levels in patients undergoing cardiopulmonary bypass and receiving high-dose aprotinin therapy indicate reduction of preoperative myocardial damage. J Thorac Cardiovasc Surg 1995; 109: 1164-72.

19 Brauner R, Laks H, Drinkwater D C et al. - Controlled periadventitial administration of verapamil inhibits neointimal smooth muscle cell proliferation and ameliorates vasomotor abnormalities in experimental vein bypass grafts. J Thorac Cardiovasc Surg 1997; 114: 53-63 